\title{
Mikroskopisch-optische Studien über die Wachstumsvorgänge an unter einseitigem Druck stehenden Kristallen in übersättigten Lösungen
}

\author{
(nach Versuchen mit W. Buss) ${ }^{1}$
}

\author{
Von Rudolf Mosebach \\ Aus dem Mineralogisch-Petrographischen Institut der Universität Tübingen \\ (Z. Naturforschg. 5 a, 508-513 [1950]; eingegangen am 22. August 1950)
}

\begin{abstract}
Mit Hilfe einer neuen mikroskopisch-optischen Methode werden Wachstumsvorgänge in übersättigter Lösung an Kupfervitriol-, Kaliumbichromat- und Roten Blutlaugensalzkristallen, die unter einseitigem Druck stehen, untersucht. Bisher wurden ähnliche Versuche unter Anwendung anderer Methoden nur am K-Al-Alaun qualitativ und quantitativ durchgeführt.

Auf Grund der Erfahrungen am Alaun stellten Correns und Ulich eine allgemeine, quantitative Beziehung auf: $v P=R T \ln \left(c / c_{\mathrm{s}}\right)$. Das Verhalten der drei Salze hinsichtlich dieser Beziehung wird geprüft.

Die Methode erlaubt weiterhin, das Wachstumsverhalten einseitig gedrückter Kristalle in solchen Richtungen und an den Flächen zu studieren, die normal bzw. parallel zu der Richtung der Druckbeanspruchung liegen. Das Verhalten des Kupfervitriols konnte auch in dieser Hinsicht untersucht und auf seine Deutungsmöglichkeiten hin näher diskutiert werden.
\end{abstract}

$\mathrm{I}^{\mathrm{n}}$ n neuerer Zeit bereicherten besonders Correns und Mitarbb. ${ }^{2-5}$ unsere Kenntnis von den Wachstumsvorgängen an Kristallen, die unter der Wirkung einseitigen Druckes stehen.

Correns erarbeitete im wesentlichen am K-AlAlaun die Ergebnisse, welche ihn in Zusammenarbeit mit $\mathrm{H}$. Ulich veranlaßten, eine quantitative Beziehung zu deren Beschreibung aufzustellen. Diese lautet:

$$
v P=R T \ln \left(c / c_{\mathrm{s}}\right),
$$

wobei $v=$ Molvolumen der Substanz, $P=$ Druck, $R=$ Gaskonstante, $T=$ abs. Temperatur, $c=$ Kon zentration der Lösung, $c_{\mathrm{s}}=$ Sättigungskonzentration ${ }^{3}$ bedeuten.

Formel (1) wird in dem Sinne diskutiert, daß bei gegebenem Verhältnis der Übersättigung der Maximaldruck bestimmt ist, den ein wachsender Kristall in seiner übersättigten Lösung auf die Unter- bzw. Auflage ausübt. In dieser Formulierung wird der Begriff des Kristallisationsoder linearen Wachstumsdruckes theoretisch gedeutet und auf bekannte physikalische Größen und Kräfte zurückgeführt. Andererseits ist bei gegebenem Druck die Übersättigungskonzentration der Mutterlauge bestimmt, in welcher der gegen Druck wachsende Kristall mit dieser koexistieren kann. Ein von $\mathrm{R} \mathrm{i} \mathrm{e} \mathrm{c} \mathrm{k} \mathrm{e}^{6,7}$, noch früher von

1 Für wertvolle Mitarbeit habe ich meiner lieben Frau, Dr. Marga E. Mosebach, herzlich zu danken.

2 C. W. Corre n s, S.-B. preuß. Akad. Wiss. physik.math. Kl. 11, 81 [1926].

3 C. W. Corre ns, Über die Messung der sog. Kristallisationskraft. (Nach Versuchen mit W.S te in born.) Fortschr. Mineral., Kristallogr., Petrogr. 23, CV [1939].
Thomson 8 aufgestellter thermodynamischer Grundsatz von der Schmelzpunktserniedrigung bzw. Löslichkeitserhöhung einseitig belasteter Kristalle in ihren Schmelzen oder Lösungen stimmt mit den experimentellen Ergebnissen von $\mathrm{C}$ or re $\mathrm{n} \mathrm{s}$ überein.

Eine von Correns konstruierte Druckwaage erlaubt es, an in übersättigter Lösung wachsenden K-Al-Alaunkristallen die Größe dieses Druckes zu messen ${ }^{4}$. Die oben wiedergegebene Beziehung stimmt danach mit den Meßergebnissen wenigstens im Bereich geringer Drucke und Übersättigungen befriedigend überein. Bei höheren Drukken und stärkeren Übersättigungen dagegen weicht die experimentell ermittelte Kurve von der theoretischen nicht unerheblich $\mathrm{ab}$, und zwar für verschiedene Kristallflächen verschieden stark, so daß wir für $\{111\}$ und $\{110\}$ gesonderte Kurven erhalten. Wird normal zu $\{100\}$ des Alauns als Auflagefläche gedrückt, so erfolgt überhaupt kein Wachstum.

Die für kristallographisch verschiedene Flächen verschiedenen Abweichungen von der theoretischen Kurve werden zweifellos von Anisotropien des Kristalles verursacht, die in Gl. (1) nicht berücksichtigt sind.

Correns zieht zur Deutung dieser Abweichungen die verschiedenen Grenzflächenspannungen zwischen Kristall und Auf- bzw. Unterlage $\left(\sigma_{\mathrm{K}, \mathrm{U}}\right)$, KristallLösung $\left(\sigma_{\mathrm{K}, \mathrm{L}}\right)$ und Lösung-Auf- bzw. Unterlage

${ }^{4}$ C. W. Correns, Disc. Farad. Soc. 5, 267-271 [1949].

5 C. W. Correns u. W. S te in born, Z. Kristallogr. Mineral. Petrogr., Abt. A 101, 117-133 [1939].

${ }_{6}$ E. R i e ck e, Nachr. Ges. Wiss. Göttingen, math.physik. Kl. 4, 278-284 [1894].

7 E. R i e cke, Zbl. Mineral. 97-104 [1912].

8 Th o m s o n, Philos. Mag. 24, 395 [1862]. 
$\left(\sigma_{\mathrm{L}, \mathrm{U}}\right)$ heran und stellt im Anschluß an des Coudres die Beziehung auf:

$$
\sigma_{\mathrm{K}, \mathrm{U}} \geqq \sigma_{\mathrm{K}, \mathrm{L}}+\sigma_{\mathrm{L}, \mathrm{U}} \cdot
$$

Nur wenn diese Beziehung erfüllt ist, wird Lösung zwischen Kristall und Auf- bzw. Unterlage hineingezogen, der Kristall kann mithin wachsen. Weiterhin führt Correns die Abweichungen von der theoretischen Kurve auf die Verschiedenheit der Grenzflächenspannungen an verschiedenen Flächen des Alauns zurück. Sonst müßte eine strikte Proportionalität zwischen der Übersättigung $c / c_{\mathrm{s}}$ und $P$ herrschen. Um den Einfluß der Grenzflächenkräfte zu verdeutlichen, werden die Versuche in der Richtung erweitert, daß anstatt Glas als Auf- und Unterlage Glimmer- und Gipsplatten Verwendung finden.

Die Gültigkeit der Gl. (1) für andere Kristallarten als Alaun ist bisher kaum geprüft worden. Lediglich bei Correns und $\mathrm{Ste}$ inborn ${ }^{5}$ findet sich die Notiz, daß hexaedrische Kristalle von Natriumchlorat in übersättigter Lösung weder zwischen Glas-, noch zwischen Glimmer- oder Gipsplatten wachsen.

Unsere kürzlich entwickelte Methode zur Messung des Kristallwachstums ${ }^{9}$ läßt sich durch einfache Zusatzapparaturen dahin erweitern, daß auch das Wachsen einseitig gedrückter Kristalle in übersättigten Lösungen sowohl in Richtung des Belastungsdruckes als auch senkrecht dazu studiert werden kann. Die Versuche wurden an Kupfervitriol (triklin-pinakoidal), Kaliumbichromat (triklin-asymmetrisch) und Rotem Blutlaugensalz (monoklin-prismatisch) durchgeführt. Sie sollen zur Prüfung der Gl. (1) dienen und zugleich unsere Kenntnis von der Kristallisation unter einseitigem Druck auf andere Substanzen ausdehnen.

Als Kristallisationsgefäß dient eine Glasschale, deren Boden aus etwa $5 \mathrm{~mm}$ dickem Spiegelglas besteht. Die Seitenwand wurde durch Aufkitten eines Glasrings angebracht. Dieses Gefäß wird auf eine kreisförmige, eiserne Grundplatte gestellt, in deren Mitte eine Öffnung geschnitten ist. Auf der Grundplatte befinden sich 3 im Kreis stehende Führungszapfen, längs denen ein Gewicht von $780 \mathrm{~g}$ auf und ab bewegt werden kann. Das Gewicht, mit einer Zentralbohrung versehen, kann durch geeignete Auflagen noch vergrößert werden.

In der Grundplatte wird nun das Kristallisationsgefäß auf den Objekttisch eines Polarisationsmikroskopes gesetzt, der zu untersuchende, planparallele Kristall auf den Spiegelglasboden gelegt und mit einer quadratischen Glasplatte von etwa $5 \mathrm{~mm}$ Dicke bedeckt. Nach Aufbringen des Gewichtes wird schließlich die übersättigte Lösung hinzugegossen.

9 R. M o s e b a c h, Z. Naturforschg. 5a, 504 [1950].

10 D'Ans-Lax, Taschenbuch für Chemiker und Physiker. Berlin 1943.
Die gesamte weitere Beobachtung, Messung und Auswertung erfolgt wie kürzlich ${ }^{9}$ beschrieben. Blindversuche mit neutralen Flüssigkeiten (Glycerin) wiesen nach, daß Verfälschungen etwa infolge Erwärmung durch das kurz brennende Bogenlicht oder durch Spannungsdoppelbrechung von Glasteilen der Apparatur nicht in Erscheinung treten. Letztere würde wahrscheinlich nicht sehr störend wirken, da sie als im wesentlichen konstant bleibend beim Bilden der Gangunterschiedsdifferenzen eliminiert würde.

Die Variation des Belastungsdruckes wurde bei konstant gehaltenem Gewicht durch passende Größenbemessung der Kristallplättchenoberfläche erzielt. Sie ließ sich unter dem Mikroskop mit Hilfe eines Okularmikrometers genügend genau ermitteln. Der Druck, auf $\mathrm{kg} / \mathrm{qcm}$ umgerechnet, dürfte der Genauigkeit von $\pm 2 \mathrm{~kg} / \mathrm{qcm}$ im ungünstigsten Fall entsprechen. Die Versuche führten wir ausnahmslos nachts in einem auf $1-2^{\circ} \mathrm{C}$ thermokonstanten Raum aus: Ein Rühren der übersättigten Lösungen war bei der geringen Menge von 15-20 $\mathrm{cm}$ und der Kristallplättchendicke von $0,1-0,5 \mathrm{~mm}$ nicht notwendig. Nach den Versuchen zeigten die Kriställchen im Mikroskop weder stufen- oder treppenförmiges Wachstum noch etwa Anzeichen von Vizinalfächenbildung.

Durch Konstruktion einer geeigneten Spannvorrichtung konnte weiter auch das Verhalten einseitig belasteter Kristalle in übersättigter Lösung an Flächen beobachtet werden, die etwa parallel zur Druckrichtung liegen. Die Vorrichtung überträgt mittels geeichter Federn einen Druck auf 2 künstlich erzeugte Flächen, die etwa senkrecht zu den Flächen liegen, die beobachtet werden sollen. $\{1 \overline{1} \overline{1}\}$, $\{110\}$ und $\{100\}$ des Kupfervitriols wurden so geprüft. Eichversuche ergaben bei dieser Druckübertragung einen Fehler von höchstens $\pm 2 \mathrm{~kg} / \mathrm{qcm}$.

Bei dieser Untersuchung erwies es sich als schwierig, in der Dimensionierung des Kriställchens unter eine Kantenlänge von etwa $2 \mathrm{~mm}$ hinunterzugehen. Das Kriställchen selbst muß dabei etwa Würfelform haben. Es liegt während des Versuches samt der Spannvorrichtung in der Kristallisierschale, umgeben von der übersättigten Lösung. Die parallel zur Druckrichtung liegenden, beobachtbaren Flächen liegen dabei in freiem Kontakt mit der Mutter-

\begin{tabular}{|c|c|c|c|}
\hline & $\mathrm{CuSO}_{4} \cdot 5 \mathrm{H}_{2} \mathrm{O}$ & $\mathrm{K}_{2} \mathrm{Cr}_{2} \mathrm{O}_{7}$ & $\mathrm{~K}_{3} \mathrm{Fe}(\mathrm{CN})_{6}$ \\
\hline $\begin{array}{c}\text { Mol.-Gew. } \\
\text { Dichte }\end{array}$ & 249,72 & 294,2 & 329,26 \\
Molvolumen & 2,28 & 2,69 & 1,89 \\
Temperatur & 109,53 & 109,37 & 174,21 \\
abs. & 291,19 & 294,19 & 294,19 \\
\hline$c / c_{\mathrm{s}}$ & \multicolumn{3}{|c|}{$P$ in $\mathrm{kg} / \mathrm{qcm}$} \\
\hline \multicolumn{3}{|c|}{} \\
\hline 1,05 & 10,99 & 11,12 & 6,98 \\
1,10 & 21,47 & 21,73 & 13,64 \\
1,15 & 31,49 & 31,86 & 20,00 \\
1,20 & 41,08 & 41,56 & 26,09 \\
1,25 & 50,28 & 50,87 & 31,94 \\
1,30 & 59,11 & 59,81 & 37,55 \\
1,35 & 67,62 & 68,71 & 42,95 \\
1,40 & 75,82 & 76,71 & 48,16 \\
\hline
\end{tabular}

Tab. 1. 


\begin{tabular}{|c|c|c|c|c|c|c|c|c|}
\hline $\begin{array}{l}\text { Versuch } \\
\text { Nr. }\end{array}$ & Fläche & $\begin{array}{l}\text { Versuchs- } \\
\text { dauer in } \\
\text { Stdn. }\end{array}$ & $c / c_{\mathrm{s}}$ & $\begin{array}{c}\text { Druck } \\
\text { in } \mathrm{kg} / \mathrm{qcm}\end{array}$ & $\begin{array}{c}\Delta \Gamma_{1} \\
\text { in } \mathrm{m} \mu\end{array}$ & $\begin{array}{l}\Delta \Gamma_{2} \\
\text { in m } \mu\end{array}$ & $\begin{array}{l}\text { Wachstum in } \\
\mu \text { während d. } \\
\text { Vers.-Dauer }\end{array}$ & $\begin{array}{l}\text { Wachstum } \\
\text { in } \mu \text { pro Tag }\end{array}$ \\
\hline 1 & $\{110\}$ & $12^{1 / 2}$ & 1,06 & 11,5 & 33 & 30 & 2,1 & 4,0 \\
\hline 2 & " & $8^{1 / 4}$ & 1,08 & 16,3 & 20 & 24 & 1,4 & 4,0 \\
\hline 6 & $"$ & $9^{1 / 4}$ & - 1,21 & 35,4 & 11 & 13 & 0,8 & 2,6 \\
\hline 8 & $"$ & $10^{3 / 4}$ & 1,45 & 47,1 & 10 & 13 & 0,8 & 2,1 \\
\hline 10 & $"$ & 10 & 1,40 & 46,2 & 14 & 17 & 1,0 & 2,4 \\
\hline 11 & $"$ & $9^{3 / 4}$ & 1,36 & 46,2 & 8 & 10 & 0,6 & 1,4 \\
\hline 14 & $"$ & 11 & 1,27 & 44,3 & 8 & 11 & 0,7 & 1,5 \\
\hline 15 & ” & $9^{1 / 4}$ & 1,28 & 40,5 & 12 & 14 & 0,8 & 2,6 \\
\hline 16 & $"$ & $8^{1 / 2}$ & 1,25 & 40,5 & 9 & 13 & 0,7 & 2,0 \\
\hline 21 & $"$ & $9^{1 / 2}$ & 1,14 & 28,8 & 18 & 23 & 1,4 & 3,7 \\
\hline 23 & $"$ & $8^{3 / 4}$ & 1,12 & 23,0 & 20 & 24 & 1,4 & 3,7 \\
\hline 25 & $\{100\}$ & 12 & 1,07 & 12,8 & 33 & 39 & 1,4 & 2,8 \\
\hline 29 & $"$ & 8 & 1,14 & 15,5 & 15 & 17 & 0,6 & 1,8 \\
\hline 30 & $"$ & 9 & 1,40 & 37,2 & 10 & 11 & 0,4 & 1,1 \\
\hline 31 & $"$ & $14^{3} / 4$ & 1,35 & 37,2 & 31 & 34 & 1,3 & 2,1 \\
\hline 32 & $"$ & 9 & 1,30 & 36,5 & 12 & 13 & 0,5 & 1,3 \\
\hline 37 & $"$ & $10^{1 / 2}$ & 1,40 & 41,2 & 12 & 9 & 0,4 & 0,9 \\
\hline 39 & $"$ & $9^{1 / 4}$ & 1,24 & 30,6 & 11 & 14 & 0,5 & 1,3 \\
\hline 41 & ” & $8^{1 / 4}$ & 1,19 & 26,3 & 12 & 14 & 0,5 & 1,5 \\
\hline 44 & $"$ & $8^{1 / 4}$ & 1,09 & 17,8 & 10 & 13 & 0,5 & 1,5 \\
\hline 46 & $\{1 \overline{1} \overline{1}\}$ & $8^{3 / 4}$ & 1,05 & 10,3 & 28 & 31 & 1,4 & 3,7 \\
\hline 48 & & $9^{1 / 1}$ & 1,40 & 31,6 & 28 & 34 & 1,5 & 4,0 \\
\hline 49 & $"$ & $11^{1 / 1_{4}}$ & 1,35 & 31,6 & 32 & 37 & 1,7 & 3,6 \\
\hline 50 & $"$ & 8 & 1,30 & 31,6 & 27 & 28 & 1,3 & 3,9 \\
\hline 53 & $"$ & $9^{3}{ }_{4}$ & 1,23 & 22,8 & 31 & 27 & 1,4 & 3,4 \\
\hline 54 & $"$ & $10^{1 / 2}$ & 1,19 & 22,8 & 31 & 32 & 1,5 & 3,4 \\
\hline 58 & $"$ & $9^{3 / 4}$ & 1,40 & 33,9 & 22 & 29 & 1,3 & 3,1 \\
\hline 59 & $"$ & 12 & 1,45 & 35,2 & 10 & 14 & 0,6 & 1,2 \\
\hline 61 & $"$ & 10 & 1,26 & 26,8 & 32 & 35 & 1,6 & 3,8 \\
\hline 64 & $"$ & $11^{1 / 4}$ & 1,13 & 18,5 & 48 & 46 & 2,3 & 5,0 \\
\hline 67 & $”$ & $10^{1 / 2}$ & 1,10 & 14,2 & 47 & 46 & 2,3 & 5,3 \\
\hline
\end{tabular}

Tab. 2. Kupfervitriol, Flächen normal zur Druckrichtung orientiert. Temperatur $18^{\circ} \mathrm{C}$.

lauge und erlauben eine einwandfreie mikroskopisch-kompensatorische Prüfung ihres Wachstums.

Bei Wachstum unter einseitigem Druck kann der Schichtdickenzuwachs mindestens angenähert bestimmt werden. Es ist zwar nicht ohne weiteres angängig, den Gangunterschiedszuwachs $\Delta \Gamma$ mit der am unbelasteten Kristall bestimmten Doppelbrechung zu reduzieren. Lassen wir aber eine Änderung der Doppelbrechung durch die Druckbelastung von $10 \% \mathrm{zu}$, dann sind die Werte des Schichtdickenzuwachses, die mit den Doppelbrechungen unbelasteter Kristalle reduziert worden sind, immer noch sehr brauchbar.

Um die Versuchsergebnisse mit der Beziehung (1) vergleichen zu können, stellen wir hier noch die notwendigen Daten und einige errechnete Punkte für die theoretischen Kurven der interessierenden Substanzen zusammen (Tab. 1).

$$
\mathrm{Kupfervitriol} \mathrm{CuSO}_{4} \cdot 5 \mathrm{H}_{2} \mathrm{O}
$$

Zur Untersuchung kamen planparallel durch $\{110\}$, $\{100\}$ und $\{1 \overline{1} \overline{1}\}$ begrenzte Kriställchen. Die nach $\{1 \overline{1} \overline{1}\}$ wurden durch Zersägen größerer Kristalle hergestellt und zum Ausheilen so lange in nur wenig übersättigte Mutterlauge gelegt, bis die mikroskopische Untersuchung einwandfrei glatte Beschaffenheit der Oberfläche zeigte. Die Richtung des Belastungsdruckes ist normal auf den Flächen. Tab. 2 sowie Abb. 1 enthalten die Ergebnisse. Aus
Gründen der Raumersparnis wurden die Versuche, bei denen kein Wachstum stattfand, in Tab. 2 nicht aufgenommen. Sie sind aus Abb. 1 zu entnehmen.

Die übersättigten Lösungen dieses Salzes konnten bisweilen 12-15 Stdn. keimfrei gehalten werden. Im allgemeinen endeten die Wachstumsversuche unter Druck nach der angegebenen Dauer durch Keimbildung von selbst, oder sie mußten, da wegen der Temperaturkonstanz des Raumes nur die Nachtstunden für die Messungen zur Verfügung standen, bei Tagesbeginn abgebrochen werden.

Zur Sicherung des Wertes für den Dickenzuwachs der Kristalle empfahl es sich wegen der relativ schwachen Doppelbrechung des Kupfervitriols, den Zuwachs $\Delta \Gamma$ an 2 Kompensationsstreifen zu messen. In den Tab. sind diese als $\Delta T_{1}$ und $\Delta T_{2}$ bezeichnet. Die Werte für das Wachstum wurden aus dem Mittel beider errechnet.

Zur Reduktion der $\Delta T$ in $\mu$ wurden die Doppelbrechungswerte $\mathcal{L}_{\{110\}}=0,0153, \Delta_{\{100\}}=0,0259$ und $J_{\{1 \overline{1} \overline{1}\}}$ $=0,02079$ eingesetzt. Es ergab sich ein sehr geringes Dickenwachstum in $\mu$ pro Tag, wobei zu berücksichtigen ist, daß die Kristalle an der Ober- und Unterfläche etwa gleichschnell wachsen, was auch für die Versuche am Kaliumbichromat zutrifft. Immerhin sind aber auch diese geringen Wachstumsbeträge durch die Kompensationsmethode sicher bestimmbar. 
Es gelingt, durch Variation der Versuchsbedingungen und die Beobachtung, ob Wachstum stattfindet oder nicht, die für jede Fläche charakteristische Kurve im Diagramm mit der Abszisse $c / c_{\mathrm{s}}$ und Ordinate $P$ einzugrenzen und mit der theoretischen Kurve Gl. (1) zu vergleichen (Abb. 1). Die Abhängigkeit der Über-

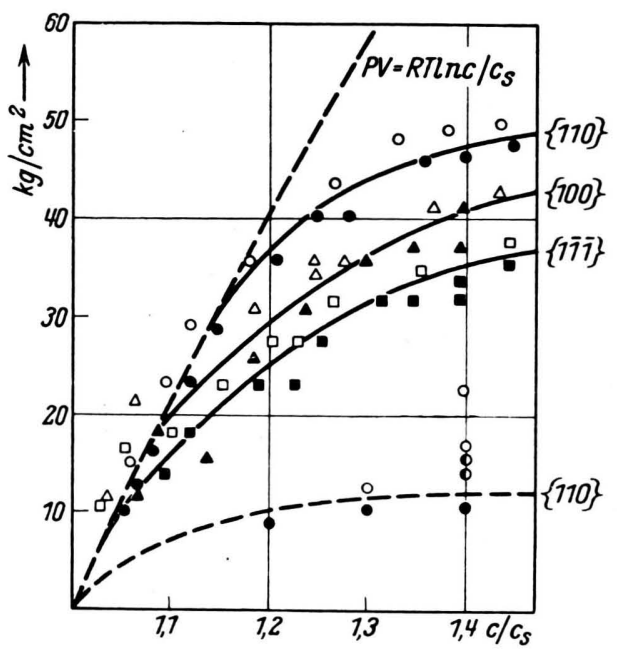

Abb. 1. Abhängigkeit der Übersättigung vom Belastungsdruck. Kupfervitriol $18^{\circ} \mathrm{C}$, nicht gerührte Lösung. Die 3 ausgezogenen Kurven entsprechen den Flächen in Normallage zur Druckrichtung. Die Indices sind jeweils rechts am Rande der Abb. angeschrieben. Ausgefüllte Signaturen (Kreise, Dreiecke und Quadrate) bedeuten Wachstum, leere Signaturen kein Wachstum. Kreise für $\{110\}$, Dreiecke für $\{100\}$ und Quadrate für $\{1 \overline{1} \overline{1}\}$. Die unterste, gestrichelte Kurve stellt die Verhältnisse für $\{110\}$ dar, wenn die Fläche parallel zur Druckrichtung am deformierten Kristall liegt. Halb ausgefüllte Kreise: Nur geringfügiges und vorübergehendes Wachstum.

sättigung vom Belastungsdruck bei Kupfervitriol ähnelt der beim Alaun, am besten für $\{110\}$. Der Kurvenverlauf differiert mehr für $\{100\}$, am meisten für $\{1 \overline{1} \overline{1}\}$. Die experimentellen Kurven der Abb. 1 finden etwa bei $P=57 \mathrm{~kg} / \mathrm{qcm}$ ihr Ende, da bei diesem Druck die Grenze der Bruchfestigkeit von Kupfervitriol liegt.

$$
\mathrm{Kaliumbichromat} \mathrm{K}_{2} \mathrm{Cr}_{2} \mathrm{O}_{7}
$$

Planparallele Kriställchen, begrenzt durch Spaltflächen nach (001), (010) und (100). Druckrichtung normal zur jeweils untersuchten Fläche. Die starke Doppelbrechung des Kaliumbichromates erforderte Plättchendicken zwischen 0,1 und $0,2 \mathrm{~mm}$, die Größe der gedrückten Fläche schwankte zwischen 3 und 5 qmm. Die übersättigten Kaliumbichromatlösungen zeigen stärkere Neigung zur Keimbildung, weswegen es kaum gelang, die Versuche länger als $6 \mathrm{Stdn}$. auszudehnen. Wegen der im allgemeinen stärkeren Doppelbrechung genügte die Einstellung eines Kompensations- streifens zur Bestimmung von $\Delta \Gamma$. Dieses reduzieren wir zur Gewinnung des Wachstums in $\mu$ mit $\Delta_{(001)}=0,0128$, $\Delta_{(100)}=0,0952$ und $\Delta_{(010)}=0,07639$.

Die Versuchsergebnisse zeigt Abb. 2. Um Raum zu sparen, wurde darauf verzichtet, diese ebenso wie diejenigen des später beschriebenen Roten Blutlaugensalzes auch tabellarisch zusammenzustellen.

Der Dickenzuwachs der drei Flächen, bezogen auf das Wachstum der Ober- und Unterfläche, schwankt zwischen 2,4 und $10 \mu$ pro Tag. Die durch die Versuche eingegrenzten Kurven der Abb. 2 zeigen einen ähnlichen Verlauf wie die des Kupfervitriols. Auch hier wachsen alle drei Flächen bei geeigneten DruckKonzentrationsbedingungen.

Der Dickenzuwachs der Spaltplättchen nach (010) und (100) wurde durch Bestimmung von $\Delta T$ jeweils nach 2 Stdn. bestimmt. Die Wachstumsbeträge schwanken stärker als bei dem früher beschriebenen Wachstum ohne Belastung ${ }^{9}$. Die errechneten Tagesmittel dagegen sind sich z. Tl. sehr ähnlich. An diesen Unregelmäßigkeiten mögen neben den Grenzen der Einstellgenauigkeit des Kompensators geringe Temperaturschwankungen, vor allem aber Diffusionsverschiedenheiten beteiligt sein. Die häufig geringeren

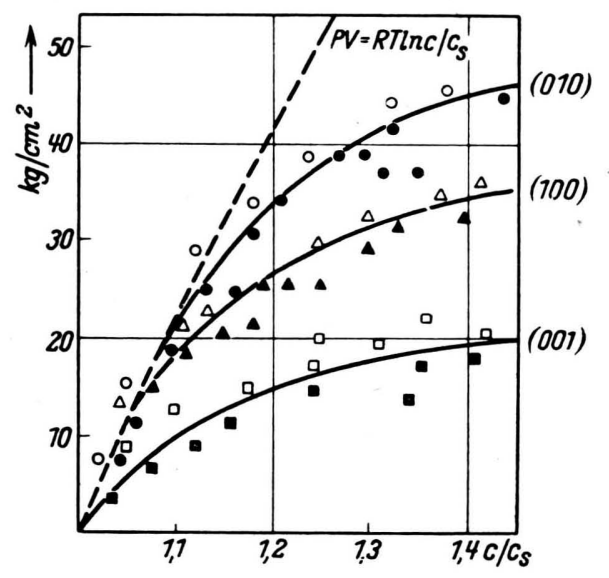

Abb. 2. Abhängigkeit der Übersättigung vom Belastungsdruck. Kaliumbichromat $21^{\circ} \mathrm{C}$, nicht gerührte Lösung. Kurven (010), (100) und (001) für Normallage der Flächen zur Druckrichtung. Im übrigen entsprechen die Signaturen denen der Abb. 1.

Wachstumsbeträge während der 4 . bis 6 . Stunde der Versuche können außerdem beginnende Keimbildung anzeigen.

An der Fläche (010) wurden einige Versuche unter Belastung durchgeführt, um zu ermitteln, ob in der ungerührten Lösung das Wachstum an der Ober- und Unterfläche des wachsenden Kristallplättchens gleichmäßig erfolgt. Tab. 3 zeigt die Resultate. Die Wachstumsbeträge der Versuche mit einer aufgekitteten Ober- bzw. Unterfläche betragen mit 4,4 und $4,0 \mu$ pro Tag fast genau die Hälfte des doppelseitigen Wachstums unter sonst gleichen 


\begin{tabular}{|c|c|c|c|c|c|c|c|}
\hline \multirow{2}{*}{$\begin{array}{c}\text { Versuch } \\
\mathrm{Nr} .\end{array}$} & \multirow{2}{*}{$\begin{array}{l}\text { Druck in } \\
\mathrm{kg} / \mathrm{qcm}\end{array}$} & \multicolumn{4}{|c|}{$\begin{array}{l}\text { Dickenzuwachs, gemessen als } \Delta T \text { in } \mathrm{m} \mu \text { (in Klammern } \\
\text { hieraus berechnet } \Delta d \text { in } \mu \text { ) während der Stde. }\end{array}$} & \multirow{2}{*}{$\begin{array}{l}\text { Wachstum in } \\
\mu \text { pro Tag }\end{array}$} & \multirow{2}{*}{ Bemerkungen } \\
\hline & & $0 .-2$. & 2.-4. & 4.-6. & insgesamt & & \\
\hline 155 & 18,7 & $23(0,30)$ & $25(0,33)$ & $34(0,45)$ & $82(1,08)$ & 4,4 & Oberseite aufgekittet \\
\hline 156 & 18,5 & $25(0,33)$ & $21(0,28)$ & $27(0,35)$ & $73(0,96)$ & 4,0 & Oberseite aufgekittet \\
\hline 157 & 19,2 & $23(0,30)$ & $25(0,33)$ & $25(0,33)$ & $73(0,96)$ & 4,0 & Unterseite aufgekittet \\
\hline 158 & 19,4 & $22(0,29)$ & $24(0,32)$ & $29(0,38)$ & $75(0,99)$ & 4,0 & Unterseite aufgekittet \\
\hline 118 & 19,2 & $50(0,66)$ & $55(0,72)$ & $51(0,67)$ & $156(2,05)$ & 8,2 & $\begin{array}{l}\text { Flächenwachstum } \\
\text { beiderseits }\end{array}$ \\
\hline
\end{tabular}

Tab. 3. Kaliumbichromat. (010) normal zur Druckrichtung. Temperatur $21^{\circ} \mathrm{C}, c / c_{\mathrm{s}}=1,10$.

Bedingungen. Auch hierdurch wird bestätigt, daß der Dickenzuwachs der Kristalle planparallel erfolgt und gleichmäßig an Ober- und Unterfläche, obgleich die Lösung nicht gerührt wird.

Der durch die triklin-pediale Symmetrië bedingte Unterschied der beiden Begrenzungsflächen der Spaltplättchen tritt auch hier, wohl infolge des langsamen Wachstums und der relativ geringen Übersättigung, nicht in Erscheinung.

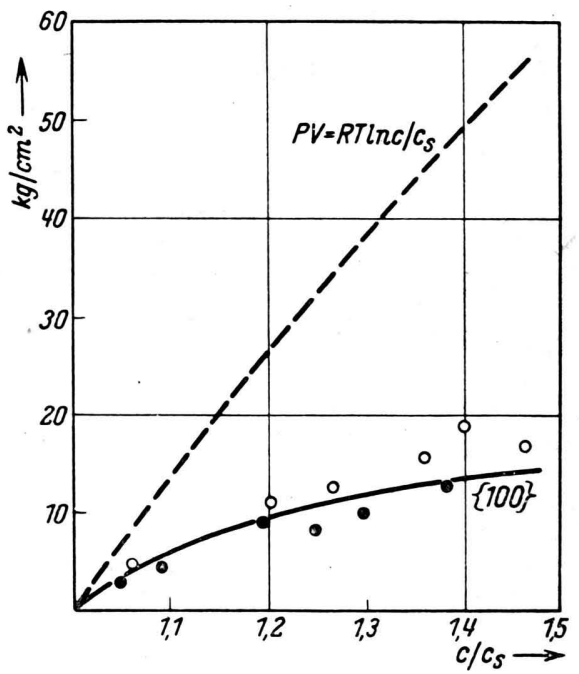

Abb. 3. Abhängigkeit der Übersättigung vom Belastungsdruck. Rotes Blutlaugensalz $21^{\circ} \mathrm{C}$, nicht gerührte Lösung. Normallage von $\{100\}$ zur Druckrichtung. Ausgefüllte

Kreise: Wachstum; leere Kreise: Kein Wachstum.

\section{Rotes Blutlaugensalz $\mathrm{K}_{3} \mathrm{Fe}(\mathrm{CN})_{6}$}

Planparallele Plättchen, begrenzt durch die Spaltfläche $\{100\}$. Nach Groth 11 errechnet sich $\Delta_{\{100\}}=0,0142$. Der Dickenzuwachs ist unter sonst gleichen Versuchsbedingungen bei Rotem Blutlaugensalz etwas größer mit 10,0 und $16 \mu$ pro Tag. Abb. 3 läßt ein stärkeres Differieren der Kurve für $\{100\}$ von der theoretischen erkennen, welches praktisch schon am Koordinatenanfangspunkt be-

11 P. Groth, Chemische Krystallographie, I, Leipzig ginnt. Natürlich ist es möglich, daß sich auch bei dieser Substanz die Kurven anderer noch nicht untersuchter Flächen besser an die errechnete Kurve anschmiegen.

$$
\begin{aligned}
& \text { Das Verhalten der Flächen }\{110\},\{100\} \\
& \text { und }\{1 \overline{1} \overline{1}\} \text { des Kupfervitriols } \\
& \text { bei Lage parallel zur Druckrichtung }
\end{aligned}
$$

Mit Hilfe der schon weiter oben beschriebenen Spannvorrichtung wurden Kristälichen so belastet, daß nacheinander die 3 bereits in Normallage zur Druckrichtung untersuchten Flächen in Parallellage mit den übersättigten Lösungen in Berührung kamen. Das Ergebnis scheint zunächst überraschend. Obwohl alle 3 Pinakoide, senkrecht zur Druckrichtung liegend, bei geeigneten Druck-Übersättigungsbedingungen wachsen können, tun dies in druckparalleler Lage $\{100\}$ und $\{1 \overline{1} \overline{1}\}$ überhaupt nicht. $\{110\}$ ist bedingt wachstumsfähig. Die quantitativen Ergebnisse zeigt Tab. 4.

Bei einer Konzentration $c / c_{\mathrm{s}}=1,4$ und $P=22 \mathrm{~kg} / \mathrm{qcm}$ sowie $15 \mathrm{~kg} / \mathrm{qcm}$ wächst auch $\{110\}$ nicht, während dieselbe Fläche, senkrecht zum Druck liegend, bei der gleichen Konzentration und Belastung von $46,2 \mathrm{~kg} / \mathrm{qcm}$ Wachstum zeigt. Bei $c / c_{\mathrm{s}}=1,4$ und $P=14 \mathrm{~kg} / \mathrm{qcm}$ beginnt $\{110\}$ zunächst zu wachsen mit $2,4 \mu$ in den beiden ersten Stunden, 1,05 $\mu$ in zwei weiteren Stunden, um dann das Wachstum einzustellen. Das gleiche zeigen 2 Versuche mit $c / c_{\mathrm{s}}=1,3$ und $P=12 \mathrm{~kg} / \mathrm{qcm}$. Das Wachstum geht mit zeitlich abnehmendem Betrag von insgesamt etwas über $2 \mu$ nur über $4 \mathrm{Stdn}$. hin und bleibt in weiteren 2 Stdn. stehen. Erst bei noch niedrigeren Belastungen scheint sich ein dauerndes seitliches Wachstum des gedrückten Kristalles einzustellen und den Wachstumsverhältnissen ähnlich $\mathrm{zu}$ werden, die für unbelastete Kristalle gelten.

Tragen wir diese Ergebnisse in Abb. 1 ein, dann hätten wir uns die Kurven für $\{100\}$ und $\{1 \overline{1} \overline{1}\}$ als mit der Abszissenachse zusammenfallend zu denken, während die Kurve für $\{110\}$ durch die unterste, gestrichelte dargestellt wird, deren Verlauf allerdings von etwa $c / c_{\mathrm{s}}=1,2$ bis 1 interpoliert ist.

Für den Bereich von Belastungsdrucken größer als etwa $10-12 \mathrm{~kg} /$ qcm sind die betrachteten Kristallflächen trotz zweifellos gehemmter Diffusion gegenüber den Flächen parallel zur Druckrichtung wachstumsbevorzugt. Erst bei Drucken unterhalb dieser 


\begin{tabular}{|c|c|c|c|c|c|c|c|}
\hline \multirow[t]{2}{*}{ Versuch Nr. } & \multirow{2}{*}{$c / c_{\mathrm{s}}$} & \multirow{2}{*}{$\begin{array}{l}\text { Druck in } \\
\mathrm{kg} / \mathrm{qcm}\end{array}$} & \multicolumn{4}{|c|}{$\begin{array}{c}\text { Dickenzuwachs, gemessen als } \Delta T \text { in } \mathrm{m} \mu \text { (in Klammern } \\
\text { hieraus berechnet } \Delta d \text { in } \mu \text { ) während der Stde. }\end{array}$} & \multirow{2}{*}{$\begin{array}{l}\text { Wachstum in } \\
\mu \text { pro Tag }\end{array}$} \\
\hline & & & $0 .-2$. & 2.-4. & 4.-6. & insgesamt & \\
\hline 191 & 1,4 & 22 & 0 & 0 & 0 & 0 & 0 \\
\hline 192 & 1,4 & 15 & 0 & 0 & 0 & 0 & 0 \\
\hline 193 & 1,4 & 14 & $37(2,41)$ & $16(1,05)$ & 0 & $53(3,46)$ & 3,46 \\
\hline 194 & 1,3 & 12 & $22(1,44)$ & $12(0,78)$ & 0 & $34(2,22)$ & 2,22 \\
\hline 195 & 1,3 & 12 & $20(1,31)$ & $13(0,85)$ & 0 & $33(2,16)$ & 2,16 \\
\hline 196 & 1,3 & 11 & $22(1,44)$ & $23(1,50)$ & $11(0,72)$ & $56(3,66)$ & 14,6 \\
\hline 197 & 1,3 & 11 & $20(1,31)$ & $30(1,96)$ & $26(1,70)$ & $76(4,97)$ & 19,9 \\
\hline 198 & 1,2 & 8 & $44(2,88)$ & $49(3,20)$ & $48(3,13)$ & $141(9,21)$ & 36,8 \\
\hline
\end{tabular}

Tab. 4. Kupfervitriol. $\{110\}$, parallel zur Druckrichtung orientiert. Temperatur $22^{\circ} \mathrm{C}$.

Grenze könnte die Diffusionsbehinderung der drucknormal gelegenen Flächen zu einer Bevorzugung der Flächen etwa parallel zur Druckrichtung führen.

Die gleichen Flächen des Kupfervitriolkristalles verhalten sich also je nach ihrer Lage normal oder parallel zur Druckrichtung verschieden. In letzterer wachsen sie trotz bester Berührung mit der Mutterlauge nicht oder nur sehr wenig, während in der diffusionsbehinderten Lage der Flächen normal zur Druckrichtung weitgehend Wachstum zu verzeichnen ist. Die Flächen eines druckdeformierten Kristalles verlieren anscheinend wachsend mit dem einseitigen Druck ihre Keimwirkung auf die übersättigte Lösung. Bei Lage der Flächen normal zur Druckrichtung bleibt sie am längsten erhalten, parallel dazu geht sie sehr bald verloren. Dies ist einleuchtend, denn die Deformation des Kristalles wirkt sich auf den druckparallelen Seitenflächen am stärksten aus, am geringsten auf den drucknormalen Auflageflächen. Jedoch ist auch für die gedrückte Fläche die Deformation nicht gleich Null, sondern nur ein Minimum. Bei höheren Drucken kann auch hier die Deformationswirkung so stark werden, daß die Impfwirkung erlischt. Suchen wir so die Ursache des gegenüber der theoretischen Kurve differierenden Verlaufes der experimentell ermittelten in den anisotropen Koeffizienten des Kristalles, die für den Deformationszustand verantwortlich sind, welchen der einseitige Druck hervorruft, dann würde die unbestreitbar vorhandene Druckproportionalität der Differenzen und andererseits die Anisotropie verständlich, die aus der
Lage der Kurven für jede kristallographisch verschiedene Fläche spricht.

Die Lage der experimentell ermittelten Kurven für die verschiedenen Flächen vom Alaun, Kupfervitriol und Kaliumbichromat, verglichen mit den Verschiebungsgeschwindigkeiten $\boldsymbol{v}_{(\mathrm{hkl})}$ der unbelasteten Kristalle, ergibt eine bemerkenswerte Tatsache: Bei dem K-Al-Alaun dürfte zweifellos die am langsamsten wachsende Fläche $\{111\}$, die schnellste $\{100\}$ sein. Dazwischen wird $\{110\}$ liegen. $\{111\}$ entspricht bei dem Alaun der Fläche, deren Kurve von der theoretischen am geringfügigsten differiert.

Das Verhältnis der Verschiebungsgeschwindigkeiten der 3 untersuchten Flächen des Kupfervitriols bei $c / c_{\mathrm{s}}=1,07$ und $22^{\circ}$ ist $v_{(110)}: v_{(100)}: v_{(111)}=1: 1,3: 2,2$. Für Kaliumbichromat gilt bei der gleichen Konzentration und derselben Temperatur $v_{(010)}: v_{(001)}: v_{(100)}$ $=1: 1,8: 3,1^{9}$. Unter der Voraussetzung, daß sich bei noch höheren UUbersättigungsgraden das Verhältnis dieser $v_{(\mathrm{hkl})}$ nicht wesentlich ändert, d. h. die Reihenfolge vertauscht wird, 'zeigt sich auch bei unseren Substanzen die gleiche Tatsache: Die Flächen geringster Verschiebungsgeschwindigkeit sind auch diejenigen, deren experimentell ermittelte Kurve am wenigsten von der theoretischen abweicht. Diese Proportionalität gilt für alle Flächen. $\mathrm{Ob}$ dieser Sachverhalt einer Regel oder einer Gesetzmäßigkeit entspricht, läßt sich bei der geringen $\mathrm{Zahl}$ von drei nun einigermaßen bekannten Beispielen noch nicht beurteilen. Dazu bedarf es der Kenntnis weitaus größerer Mengen experimentell gesicherter Befunde. 